\title{
LARGE DEVIATIONS FOR DYNAMICAL SYSTEMS WITH STRETCHED EXPONENTIAL DECAY OF CORRELATIONS
}

\author{
ROMAIN AIMINO AND JORGE MILHAZES FREITAS
}

\begin{abstract}
We obtain large deviations estimates for systems with stretched exponential decay of correlations, which improve the ones previously obtained in the literature. As a consequence we obtain better large deviations estimates for Viana maps and get large deviations estimates for a class of intermittent maps with stretched exponential loss of memory.
\end{abstract}

\section{INTRODUCTION}

In the last two decades the study of statistical properties of non-uniformly dynamical systems has been capturing much interest and attention. The memory loss of the systems given in terms of decay of correlations and its connections with limiting laws, such as central limit theorems, invariance principles, extreme value distributions, and other properties such as large deviation principles have been investigated thoroughly. In order to prove such properties, several different techniques have been used, such as: inducing, coupling, spectral analysis of transfer operators or renewal equations. One of the tools that has revealed to be very powerful is the construction of Gibbs-Markov structures called Young towers whose inducing times allow to describe the rates of mixing of the system (YYou98, You99]) and ultimately obtain estimates for large deviations ([MN08]) and several types of limiting laws.

In [AFLV11, the authors studied the relations between the rates of decay of correlations, large deviation estimates and the tail of the inducing times of Young towers. In particular, they proved a sort of reciprocal of Young's results, namely, they have shown that if the system has a certain rate of decay of correlations, then it admits a Young tower whose inducing times' tail decays at a similar rate. This was done both for local diffeomorphisms and maps with critical/singular sets. The construction of the Young tower and the estimates on the induced time tail follow from large deviations estimates for the expansion function and for the distance to the critical/singular set. Hence, at the core of that paper is a result ([AFLV11, Theorem D]) that establishes a connection between the rates for large deviations

RA was partially supported by FCT grant SFRH/BPD/123630/2016. Both authors were partially supported by FCT projects FAPESP/19805/2014, PTDC/MAT-CAL/3884/2014 and PTDC/MAT-PUR/28177/2017, with national funds, and by CMUP (UID/MAT/00144/2019), which is funded by FCT with national (MCTES) and European structural funds through the programs FEDER, under the partnership agreement PT2020. RA would like to thank Jean-René Chazottes and Cesar Maldonado for interesting discussions on this topic. JMF would like to thank José Alves for useful comments. The authors would also like to thank the referees for their useful suggestions. 
estimates of observable functions and the rates of decay of correlations of those observables against essentially bounded functions. Two types of decay rates were considered: polynomial and stretched exponential. To be more precise, this result asserted that if a systems has decay of correlations for observables on a certain Banach space against all essentially bounded functions at a polynomial rate or at a stretched exponential rate, then the large deviations estimates for those particular observables decay, respectively, at a polynomial or stretched exponential rate. Moreover, the dependence of the exponents and constants was clearly stated. We remark that the polynomial case had already been proved in [Mel09]. However, as a corollary from the stretched exponential counterpart, in AFLV11, the authors obtained, for the first time, stretched exponential estimates for the large deviations of Hölder continuous observables evaluated along the orbits of Viana maps. Under certain certain more restrictive assumptions, in [AFLV11, Theorem E], the exponential case was also covered, but the assumption on the decay of correlations was rather very strong.

The main goal of this note is to improve the large deviations estimates for the stretched exponential case obtained in [AFLV11, Theorem D]. Namely, we obtain a smaller loss in the exponent of the stretched exponential rate when one goes from decay of correlations to large deviations estimates. We apply this result to Viana maps in order to obtain the best rates of large deviations estimates for these maps available in the literature. We also apply it to some some non-uniformly expanding maps introduced in CDKM18, which result from a modification of the intermittent maps studied in [LSV99] carried out in order to produce stretched exponential decay of correlations.

The proof of the result is based on a technique introduced by Gordin that allows to write the sum of the random variables generated by the dynamics as a sum of reverse time martingale differences plus a coboundary. Then the problem is reduced to control the large deviations of the sum of martingale differences. In the polynomial case, in [Mel09], this is done using Rio's inequality. In the stretched exponential case, in [AFLV11, the Azuma-Hoeffding inequality was used, instead. Here, we use again Rio's inequality and a power series expansion of the exponential moments of the sum of martingale differences in order to improve the exponent in the large deviation estimates obtained in [AFLV11, Theorem D] .

This short paper is organised as follows. In Section 2 we state the main result and give some applications. Section 3 is dedicated to the proofs.

\section{Statement of RESUlts AND APplications}

Let $T: X \rightarrow X$ be a dynamical system with an ergodic invariant probability measure $\mu$. Let $\varphi: X \rightarrow \mathbb{R}$ be an observable with $\varphi \in L^{1}(\mu)$ and let

$$
\varphi_{n}=\sum_{k=0}^{n-1} \varphi \circ T^{k} .
$$


Birkhoff's ergodic theorem guarantees that for $\mu$-a.e. $x \in X$, time averages converge to the spatial average, i.e.,

$$
\lim _{n \rightarrow \infty} \frac{1}{n} \varphi_{n}(x)=\int \varphi d \mu .
$$

The study of Large Deviations concerns the probability of observing a deviation from the mean for Birkhoff averages, namely, for a deviation size $\varepsilon>0$, we define:

$$
\operatorname{LD}(\varepsilon, \varphi, n)=\mu\left(\left|\frac{1}{n} \varphi_{n}-\int \varphi d \mu\right|>\varepsilon\right) .
$$

Of course, Birkhoff's ergodic theorem implies that for all $\varepsilon$, we have that $\operatorname{LD}(\varepsilon, \varphi, n)$ vanishes as $n \rightarrow \infty$. Large Deviations theory concerns the rate at which this quantity goes to 0 . In the classical case of independent and identically distributed random variables with a finite exponential moment, $\operatorname{LD}(\varepsilon, \varphi, n)$ decays exponentially fast in $n$ and, in fact, one can prove a Large Deviations principle which establishes that there exists a strictly convex function $I(\varepsilon)$, vanishing only at 0 , such that

$$
\lim _{n \rightarrow \infty} \frac{1}{n} \log (\operatorname{LD}(\varepsilon, \varphi, n))=-I(\varepsilon) .
$$

In this setting the function $I$ is also called Crámer function and can be obtained from the knowledge of the distribution of random variable $\varphi$. Large Deviations principles have been proved by several authors for uniformly hyperbolic systems (see for example OP88, Kif90, Lop90, You90, Wad96]).

One of the main aspects of raised in [Mel09, AFLV11] was the intimate connection between Large Deviations and Decay of Correlations.

Definition 1 (Decay of correlations). Let $\mathcal{C}_{1}, \mathcal{C}_{2}$ denote Banach spaces of real valued measurable functions defined on $X$. We denote the correlation of non-zero functions $\phi \in \mathcal{C}_{1}$ and $\psi \in \mathcal{C}_{2}$ w.r.t. a measure $\mu$ as

$$
\operatorname{Cor}_{\mu}(\phi, \psi, n):=\frac{1}{\|\phi\|_{\mathcal{C}_{1}}\|\psi\|_{\mathcal{C}_{2}}}\left|\int \phi\left(\psi \circ T^{n}\right) \mathrm{d} \mu-\int \phi \mathrm{d} \mu \int \psi \mathrm{d} \mu\right| .
$$

We say that we have decay of correlations, w.r.t. the measure $\mu$, for observables in $\mathcal{C}_{1}$ against observables in $\mathcal{C}_{2}$ if, for every $\phi \in \mathcal{C}_{1}$ and every $\psi \in \mathcal{C}_{2}$ we have

$$
\operatorname{Cor}_{\mu}(\phi, \psi, n) \rightarrow 0, \quad \text { as } n \rightarrow \infty \text {. }
$$

We say that we have decay of correlations against $L^{1}$ observables whenever this holds for $\mathcal{C}_{2}=L^{1}(\mu)$ and $\|\psi\|_{\mathcal{C}_{2}}=\|\psi\|_{1}=\int|\psi| \mathrm{d} \mu$.

For non-uniformly hyperbolic systems, decay of correlations may not be exponential and, in those cases, the decay rate of $\operatorname{LD}(\varepsilon, \varphi, n)$ is not exponential. For example, in the intermittent case of the Manneville - Pomeau maps, it has been proved that $\operatorname{LD}(\varepsilon, \varphi, n)$ decays polynomially fast (see for example [MN08, PS09, Mel09, AFLV11]). In some cases, like for Viana maps, for which streched exponential decay of correlations has been proved, the rate of decay 
of $\operatorname{LD}(\varepsilon, \varphi, n)$ is stretched exponential ([AFLV11]). For other results regarding Large Deviations for non-uniformly hyperbolic systems, see for example: AP06, MN08, Mel09, PS09, AFLV11, Var12, CTY17].

Exponential decay of correlations alone has not proved to be enough to prove exponential Large Deviations. So far, exponential Large Deviations rates have only been obtained by requiring some stronger properties, like a spectral gap for the Perron-Frobenius operator or decay of correlations against all $L^{1}$ observations (see discussion in [AFLV11]). There is some loss in the exponents when one goes from decay of correlations estimates to rates of Large Deviations, unless some more information is known.

Very recently, in [NT19], the authors show that even for very well behaved systems with exponential decay of correlations, for unbounded observables, one cannot obtain Large Deviations rates better than stretched exponential. We consider observables in $L^{\infty}$ and we managed to improve the loss in the exponents observed from the rates of decay of correlations to Large Deviations rates. However, it remains an open question whether the loss we managed to improve here is optimal (as in the unbounded case) or not.

We are now ready to state our main result. In what follows, for notational simplicity, we assume without loss of generality that $\int_{X} \varphi d \mu=0$.

Theorem 2. Let $\varphi \in L^{\infty}(\mu)$. Suppose that there exist $C_{\varphi}>0, \tau>0$ and $\theta \in(0,1]$ such that

$$
\left|\int_{X} \varphi \cdot \psi \circ T^{n} d \mu\right| \leq C_{\varphi}\|\psi\|_{L_{\mu}^{\infty}} e^{-\tau n^{\theta}}, \forall \psi \in L^{\infty}(\mu), n \geq 0 .
$$

Then there exists $c=c(\theta, \tau)>0$ such that for all $n \geq 1$ and $\epsilon>0$,

$$
\mu\left(\left|\varphi_{n}\right|>n \epsilon\right) \leq 2 e^{-\tau^{\prime} \epsilon^{2 \theta^{\prime}} n^{\theta^{\prime}}},
$$

with $\theta^{\prime}=\frac{\theta}{\theta+1}, \tau^{\prime}=c \widetilde{C}_{\varphi}^{-2 \theta^{\prime}}$ and $\widetilde{C}_{\varphi}=\max \left\{\|\varphi\|_{L_{\mu}^{\infty}}, C_{\varphi}\right\}$.

Note that the exponent $\theta^{\prime}=\frac{\theta}{\theta+1}$ that we obtain for the large deviations estimate is larger than the one obtained in [AFLV11, Theorem D], where the exponent was equal to $\frac{\theta}{\theta+2}$.

Of course, this result gives rise to the natural question regarding to which extent the exponent for the stretched exponential large deviations estimate that we managed to improve here is optimal.

2.1. Large deviations for Viana maps. In [Via97, Viana introduced an important class of nonuniform expanding dynamical systems with critical sets in dimension greater than one. This class of maps can be described as follows. Let $a_{0} \in(1,2)$ be such that the critical point $x=0$ is pre-periodic for the quadratic map $Q(x)=a_{0}-x^{2}$. Let $S^{1}=\mathbb{R} / \mathbb{Z}$ and $b: S^{1} \rightarrow \mathbb{R}$ be a Morse function, for instance, $b(s)=\sin (2 \pi s)$. For fixed small $\alpha>0$, consider the map

$$
\begin{aligned}
& \hat{f}: S^{1} \times \mathbb{R} \quad \longrightarrow \quad S^{1} \times \mathbb{R} \\
& (s, x) \quad \longmapsto \quad(\hat{g}(s), \hat{q}(s, x))
\end{aligned}
$$


where $\hat{q}(s, x)=a(s)-x^{2}$ with $a(s)=a_{0}+\alpha b(s)$, and $\hat{g}$ is the uniformly expanding map of the circle defined by $\hat{g}(s)=d s(\bmod \mathbb{Z})$ for some integer $d \geq 2$. It is easy to check that for $\alpha>0$ small enough there is an interval $I \subset(-2,2)$ for which $\hat{f}\left(S^{1} \times I\right)$ is contained in the interior of $S^{1} \times I$. Hence, $S^{1} \times I$ is a forward invariant region for any map $f$ sufficiently close to $\hat{f}$ in the $C^{0}$ topology. Any such map restricted to $S^{1} \times I$ is called a Viana map. It was shown in Alv00] that Viana maps have a unique ergodic absolutely continuous invariant probability measure (acip) $\mu$. In Gou06, it was proved that every Viana map exhibits stretched exponential decay of correlations, with $\theta=1 / 2$, for Hölder continuous functions against $L^{\infty}(\mu)$ functions. Consequently, the following corollary is a direct application of Theorem 2 ,

Corollary 3. Let $f$ be a Viana map and let $\mu$ be its unique acip. Then, for every Hölder continuous observable $\varphi$ and every $\epsilon>0$, there exist $\tau=\tau(\varphi, \epsilon)>0$ and $C=C(\varphi, \epsilon)>0$ such that $\mu\left(\left|\frac{1}{n} \varphi_{n}-\int \varphi d \mu\right|>\epsilon\right) \leq C e^{-\tau n^{1 / 3}}$.

For comparison purposes, we remark that the large deviations estimate obtained in AFLV11, Theorem G] was of the order of $e^{-\tau^{\prime} n^{1 / 5}}$.

\subsection{Large deviations for intermittent maps with stretched exponential decay of} correlations. We consider the family of interval maps $f_{\gamma}:[0,1] \rightarrow[0,1]$, with $\gamma \in(0,1]$, introduced in [CDKM18, Appendix A], which result from adapting the intermittent maps studied in [LSV99] so that the contact between the graph of $f_{\beta}$ and the identity at the fixed point creates a stretched exponential fast accumulation of the pre-orbit of $1 / 2$ at 0 , which eventually is responsible for stretched exponential decay of correlations. Namely, consider that

$$
f_{\gamma}(x)=\left\{\begin{array}{ll}
x\left(1+\frac{(\log 2)^{\gamma^{-1}-1}}{|\log x|^{\gamma^{-1}-1}}\right), & x \leq 1 / 2 \\
2 x-1, & x>1 / 2
\end{array} .\right.
$$

From [CDKM18, Theorem A.1], it follows that $f_{\gamma}$ has an absolutely continuous invariant measure $\mu$ and exhibits stretched exponential decay of correlations, with $\theta=\gamma$, for Hölder continuous functions against $L^{\infty}(\mu)$ functions. Hence, as consequence of Theorem 2, we obtain:

Corollary 4. Let $f_{\gamma}$ be as in (2) and let $\mu$ be its acip. Then, for every Hölder continuous observable $\varphi$ and every $\epsilon>0$, there exist $\tau=\tau(\varphi, \epsilon)>0$ and $C=C(\varphi, \epsilon)>0$ such that $\mu\left(\left|\frac{1}{n} \varphi_{n}-\int \varphi d \mu\right|>\epsilon\right) \leq C e^{-\tau n^{\gamma /(\gamma+1)}}$.

\section{Proof}

To prove Theorem 2, we will estimate all the moments of $\varphi_{n}$. 
Lemma 5. There exists $K=K(\theta, \tau)>0$ such that for all $q>0$ and $n \geq 1$, and all $\varphi \in L^{\infty}(\mu)$ satisfying (11), we have

$$
\left(\int_{X}\left|\varphi_{n}\right|^{q} d \mu\right)^{\frac{1}{q}} \leq K \widetilde{C}_{\varphi} q^{\frac{1}{2}\left(1+\frac{1}{\theta}\right)} n^{\frac{1}{2}}
$$

Proof. In this proof, we will use $K$ to designate a generic constant whose value can change from one occurence to the other. The value of $K$ depends only on $\theta$ and $\tau$, and in particular is independent from $n, q$ and the observable $\varphi$.

We will follow closely the proof of [Mel09, Lemma 2.1], adapted to our assumption of stretched exponential decay, keeping a precise track of the dependence in $q$ of all the estimates.

Let $\mathcal{L}: L^{1}(\mu) \rightarrow L^{1}(\mu)$ be the transfer operator of $T$, i.e. the unique operator satisfying

$$
\int_{X} \varphi \cdot \psi \circ T d \mu=\int_{X} \mathcal{L} \varphi \cdot \psi d \mu, \forall \varphi \in L^{1}(\mu), \forall \psi \in L^{\infty}(\mu) .
$$

Since $L^{\infty}(\mu)$ is the dual of $L^{1}(\mu)$, (11) implies that $\left\|\mathcal{L}^{n} \varphi\right\|_{L_{\mu}^{1}} \leq C_{\varphi} e^{-\tau n^{\theta}}$. Hence, for all $q \geq 1$,

$$
\int_{X}\left|\mathcal{L}^{n} \varphi\right|^{q} d \mu \leq\left\|\mathcal{L}^{n} \varphi\right\|_{L_{\mu}^{\infty}}^{q-1} \int_{X}\left|\mathcal{L}^{n} \varphi\right| d \mu \leq C_{\varphi}\|\varphi\|_{L_{\mu}^{\infty}}^{q-1} e^{-\tau n^{\theta}}
$$

Defining $\chi=\sum_{n=1}^{\infty} \mathcal{L}^{n} \varphi$, we get, for all $q \geq 1$,

$$
\begin{aligned}
\|\chi\|_{L_{\mu}^{q}} \leq \sum_{n=1}^{\infty}\left\|\mathcal{L}^{n} \varphi\right\|_{L_{\mu}^{q}} \leq \sum_{n=1}^{\infty} C_{\varphi}^{\frac{1}{q}}\|\varphi\|_{L_{\mu}^{\infty}}^{1-\frac{1}{q}} e^{-\frac{\tau}{q} n^{\theta}} \leq \widetilde{C}_{\varphi} \sum_{n=1}^{\infty} e^{-\frac{\tau}{q} n^{\theta}} & \leq \widetilde{C}_{\varphi} \int_{0}^{\infty} e^{-\frac{\tau}{q} t^{\theta}} d t \\
& =\widetilde{C}_{\varphi} \frac{1}{\theta}\left(\frac{q}{\tau}\right)^{\frac{1}{\theta}} \int_{0}^{\infty} e^{-s} s^{\frac{1}{\theta}-1} d s \\
& =\widetilde{C}_{\varphi} q^{\frac{1}{\theta}} \frac{1}{\theta} \frac{1}{\tau^{\frac{1}{\theta}}} \Gamma\left(\frac{1}{\theta}\right) \\
& =K \widetilde{C}_{\varphi} q^{\frac{1}{\theta}}
\end{aligned}
$$

where we have performed the change of variables $s=\frac{\tau}{q} t^{\theta}$.

Defining $\phi=\varphi+\chi-\chi \circ T$, we also have

$$
\left(\|\phi\|_{L_{\mu}^{q}} \leq\|\varphi\|_{L_{\mu}^{q}}+2\|\chi\|_{L_{\mu}^{q}} \leq\|\varphi\|_{L_{\mu}^{\infty}}+2 K \widetilde{C}_{\varphi} q^{\frac{1}{\theta}} \leq K \widetilde{C}_{\varphi} q^{\frac{1}{\theta}}\right.
$$

It is immediate to verify that $\mathcal{L} \phi=0$, and so $\left\{\phi \circ T^{k} ; k=0,1,2, \ldots\right\}$ is a sequence of reverse martingale differences. Passing to the natural extension, we can reduce the situation to the case where $\left\{\phi \circ T^{k} ; k=0,1,2, \ldots\right\}$ is a sequence of martingale differences with respect to a filtration $\left\{\mathcal{F}_{k} ; k=0,1,2, \ldots\right\}$. 
By Rio's inequality [Rio00, Theorem 2.5], we have for all $q \geq 1$ :

$$
\left\|\varphi_{n}\right\|_{L_{\mu}^{2 q}}^{2 q} \leq\left(4 q \sum_{i=1}^{n} b_{i, n}\right)^{q}
$$

where

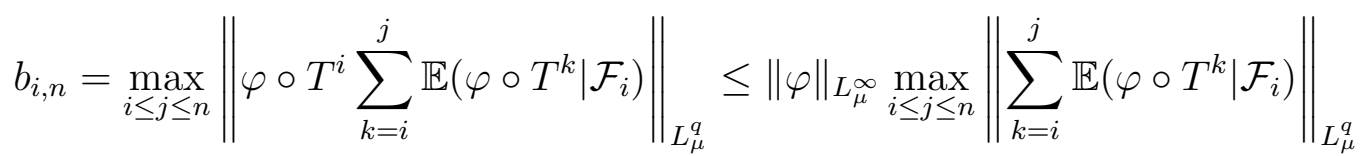

From the definition of $\phi$ and the martingale property, it follows

$$
\sum_{k=i}^{j} \mathbb{E}\left(\varphi \circ T^{k} \mid \mathcal{F}_{i}\right)=\phi \circ T^{i}-\mathbb{E}\left(\chi \circ T^{i} \mid \mathcal{F}_{i}\right)+\mathbb{E}\left(\chi \circ T^{j+1} \mid \mathcal{F}_{i}\right),
$$

so that

$$
\left\|\sum_{k=i}^{j} \mathbb{E}\left(\varphi \circ T^{k} \mid \mathcal{F}_{i}\right)\right\|_{L_{\mu}^{q}} \leq\|\phi\|_{L_{\mu}^{q}}+2\|\chi\|_{L_{\mu}^{q}} \leq K \widetilde{C}_{\varphi} q^{\frac{1}{\theta}}
$$

Hence $b_{i, n} \leq\|\varphi\|_{L_{\mu}^{\infty}} K \widetilde{C}_{\varphi} q^{\frac{1}{\theta}} \leq K \widetilde{C}_{\varphi}^{2} q^{\frac{1}{\theta}}$ and we thus obtain for all $q \geq 1$

$$
\left\|\varphi_{n}\right\|_{L_{\mu}^{2 q}}^{2 q} \leq K\left(\widetilde{C}_{\varphi}^{2} q^{1+\frac{1}{\theta}} n\right)^{q}
$$

which yields the required estimate for all $q \geq 2$. The general case of $q>0$ is deduced by changing the value of the constant $K$, since the map $q \mapsto\left(\int_{X}\left|\varphi_{n}\right|^{q} d \mu\right)^{\frac{1}{q}}$ is non decreasing for $q \in(0, \infty)$.

Lemma 6. There exists $c=c(\theta, \tau)>0$ such that for all $\varphi \in L^{\infty}(\mu)$ satisfying (1):

$$
\sup _{n \geq 1} \int_{X} e^{\tau^{\prime} n^{-\theta^{\prime}}\left|\varphi_{n}\right|^{2 \theta^{\prime}}} d \mu \leq 2
$$

with $\tau^{\prime}=c \widetilde{C}_{\varphi}^{-2 \theta^{\prime}}$.

Proof. By expanding the exponential in power series and using Fubini's theorem and Lemma 5 , we have for all $\tau^{\prime}>0$ and $n \geq 1$ :

$$
\int_{X} e^{\tau^{\prime} n^{-\theta^{\prime}}\left|\varphi_{n}\right|^{2 \theta^{\prime}}} d \mu=\sum_{k=0}^{\infty} \frac{\left(\tau^{\prime}\right)^{k} n^{-k \theta^{\prime}}}{k !} \int_{X}\left|\varphi_{n}\right|^{2 k \theta^{\prime}} d \mu \leq \sum_{k=0}^{\infty}\left(2 \theta^{\prime} \tau^{\prime} K^{2 \theta^{\prime}} \widetilde{C}_{\varphi}^{2 \theta^{\prime}}\right)^{k} \frac{k^{k}}{k !} .
$$

Since, by induction over $k$, we have $k ! \geq\left(\frac{k}{e}\right)^{k}$ for all $k \geq 1$, it follows

$$
\int_{X} e^{\tau^{\prime} n^{-\theta^{\prime}}\left|\varphi_{n}\right|^{2 \theta^{\prime}}} d \mu \leq \sum_{k=0}^{\infty}\left(2 e \theta^{\prime} K^{2 \theta^{\prime}} \widetilde{C}_{\varphi}^{2 \theta^{\prime}} \tau^{\prime}\right)^{k}=2,
$$

if we set $\tau^{\prime}=c \widetilde{C}_{\varphi}^{-2 \theta^{\prime}}$ for $c=\left(4 e \theta^{\prime} K^{2 \theta^{\prime}}\right)^{-1}$. 
Proof of Theorem 2. For all $n \geq 1$ and $\epsilon>0$, by Markov's inequality and Lemma 6,

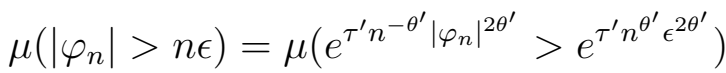

$$
\begin{aligned}
& \leq e^{-\tau^{\prime} n^{\theta^{\prime}} \epsilon^{2 \theta^{\prime}}} \int_{X} e^{\tau^{\prime} n^{-\theta^{\prime}}\left|\varphi_{n}\right|^{2 \theta^{\prime}}} d \mu \\
& \leq 2 e^{-\tau^{\prime} n^{\theta^{\prime}} \epsilon^{2 \theta^{\prime}}} \text {. }
\end{aligned}
$$

Remark 7. By changing the value of $c$, we can replace the constant 2 by any constant of the form $1+\delta, \delta>0$.

\section{REFERENCES}

[AFLV11] José F. Alves, Jorge M. Freitas, Stefano Luzzatto, and Sandro Vaienti, From rates of mixing to recurrence times via large deviations, Adv. Math. 228 (2011), no. 2, 1203-1236. MR 2822221

[Alv00] José Ferreira Alves, SRB measures for non-hyperbolic systems with multidimensional expansion, Ann. Sci. École Norm. Sup. (4) 33 (2000), no. 1, 1-32. MR 1743717 (2002i:37032)

[AP06] V. Araújo and M. J. Pacifico, Large deviations for non-uniformly expanding maps, J. Stat. Phys. 125 (2006), no. 2, 415-457. MR 2270016

[CDKM18] C. Cuny, J. Dedecker, A. Korepanov, and F. Merlevède, Rates in almost sure invariance principle for quickly mixing dynamical systems, Preprint arXiv:1811.09094, 2018.

[CTY17] Vaughn Climenhaga, Daniel J. Thompson, and Kenichiro Yamamoto, Large deviations for systems with non-uniform structure, Trans. Amer. Math. Soc. 369 (2017), no. 6, 4167-4192. MR 3624405

[Gou06] Sébastien Gouëzel, Decay of correlations for nonuniformly expanding systems, Bull. Soc. Math. France 134 (2006), no. 1, 1-31. MR MR2233699 (2008f:37066)

[Kif90] Yuri Kifer, Large deviations in dynamical systems and stochastic processes, Trans. Amer. Math. Soc. 321 (1990), no. 2, 505-524. MR 1025756

[Lop90] Artur O. Lopes, Entropy and large deviation, Nonlinearity 3 (1990), no. 2, 527-546. MR 1054587

[LSV99] Carlangelo Liverani, Benoît Saussol, and Sandro Vaienti, A probabilistic approach to intermittency, Ergodic Theory Dynam. Systems 19 (1999), no. 3, 671-685. MR MR1695915 (2000d:37029)

[Mel09] Ian Melbourne, Large and moderate deviations for slowly mixing dynamical systems, Proc. Amer. Math. Soc. 137 (2009), no. 5, 1735-1741. MR MR2470832 (2009m:37087)

[MN08] Ian Melbourne and Matthew Nicol, Large deviations for nonuniformly hyperbolic systems, Trans. Amer. Math. Soc. 360 (2008), no. 12, 6661-6676. MR MR2434305 (2009m:37086)

[NT19] Matthew Nicol and Andrew Török, A note on large deviations for unbounded observables, Preprint arXiv:1904.02227v1 arXiv:1904.02227v1, 2019.

[OP88] Steven Orey and Stephan Pelikan, Large deviation principles for stationary processes, Ann. Probab. 16 (1988), no. 4, 1481-1495. MR 958198

[PS09] Mark Pollicott and Richard Sharp, Large deviations for intermittent maps, Nonlinearity 22 (2009), no. 9, 2079-2092. MR 2534293

[Rio00] Emmanuel Rio, Théorie asymptotique des processus aléatoires faiblement dépendants, Mathématiques \& Applications (Berlin) [Mathematics \& Applications], vol. 31, Springer-Verlag, Berlin, 2000. MR 2117923

[Var12] Paulo Varandas, Non-uniform specification and large deviations for weak Gibbs measures, J. Stat. Phys. 146 (2012), no. 2, 330-358. MR 2873016 
[Via97] Marcelo Viana, Multidimensional nonhyperbolic attractors, Inst. Hautes Études Sci. Publ. Math. (1997), no. 85, 63-96. MR MR1471866 (98j:58073)

[Wad96] Simon Waddington, Large deviation asymptotics for Anosov flows, Ann. Inst. H. Poincaré Anal. Non Linéaire 13 (1996), no. 4, 445-484. MR 1404318

[You90] Lai-Sang Young, Large deviations in dynamical systems, Trans. Amer. Math. Soc. 318 (1990), no. 2, 525-543. MR MR975689 (90g:58069)

[You98] Statistical properties of dynamical systems with some hyperbolicity, Ann. of Math. (2) 147 (1998), no. 3, 585-650. MR MR1637655 (99h:58140)

[You99] , Recurrence times and rates of mixing, Israel J. Math. 110 (1999), 153-188. MR MR1750438 (2001j:37062)

Romain Aimino, Centro de Matemática da Universidade do Porto, Rua do Campo Alegre 687, 4169-007 Porto, Portugal

E-mail address: romain.aimino@fc.up.pt

$U R L:$ http://www.fc.up.pt/pessoas/romain.aimino/

Jorge Milhazes Freitas, Centro de Matemática \& Faculdade de CiÊncias da Universidade do Porto, Rua do Campo Alegre 687, 4169-007 Porto, Portugal

E-mail address: jmfreita@fc.up.pt

$U R L:$ http://www.fc.up.pt/pessoas/jmfreita/ 